\title{
Analysis and Research on Important Microorganisms in Liquor
}

\author{
Zhang Zhiwei, Wang Wei* \\ Qilu University of Technology (Shandong Academy Of Sciences), Shandong, Jinan, 250353
}

Keywords: liquor; microorganism; fermentation; fragrance

\begin{abstract}
Based on the induction and combing of related literatures, this paper discusses the research progress of important microorganisms in liquor based on the characteristics of fragrant, strong-fragrance and sauce-flavor liquor, and prospects the research methods and analysis paths of liquor microbes to explore the essence of microbial fermentation of Chinese liquor.
\end{abstract}

The important microorganisms in liquor are the general term for microorganisms that play a critical role in the production and fermentation process. They not only cover natural microorganisms, but also contain important microorganisms that have been artificially selected ${ }^{[1]}$. Specifically, it includes all microorganisms attached to raw materials, air, water, mud, kanji, production tools, in the process of liquor production. In recent years, many notable achievements have been made in the research of important microorganisms in liquor. In this paper, the research results of important microorganisms in liquor are summarized and combed based on the fragrance type, which provides a research base for exploring the nature of microbial fermentation of Chinese liquor.

\section{Microbial analysis of strong-fragrance liquor}

Liquor, wine cellar, and mashed mud are the three major areas of strong-fragrance liquor microbes. In the study of liquor, Tang Yuming and other researchers based on the correlation between air microbes and liquor quality in different areas of Daqu fermentation, the results show that the quality of liquor is more influenced by yeast and bacteria, and the source of microorganisms in liquor is mainly raw materials and water. It is mainly yeast and mold, in which bacteria also occupy a certain proportion, especially high-temperature bacteria are rich. Among the various types of bacteria, there are a lot of Bacillus. In the research of microbial research in wine cellar, the research mainly focuses on the microbial fermentation rules and the relationship between biological group and material change in the fermentation process, and on this basis, studies the incentives for the production of liquor. Xiong Changxu's research results are linked to the rules of microbial fermentation and the relationship between material changes. Gao Ling is mainly concerned with the change of microbial biomass in strong-fragrance liquor under the conditions of different Daqu additions. The results show that there is a certain correlation between the quantity relationship of microbes and the fermentation time, and the amount of the matrix does not change when it exceeds a certain proportion. And it shows the relevance. Related researchers have carried out in-depth research on the dynamic changes of microbial fermentation and the changes of logistic relationship with the fermentation process of Wuliangye ${ }^{[2]}$.

Muddy microorganisms mainly analyze the microbial groups and their deductive changes in the micro-environment of the mud, and the researchers also analyzed and explored the fields of microbial metabolism patterns and identification and separation. In the microbial microenvironment research, the main analysis and discussion of the changes in the number of microorganisms in the fermentation microenvironment, and in-depth analysis of its fauna, the results show that the aerobic and anaerobic bacteria in the mud have regularity along the fermentation process. Variety, Tang Jianmin's research uses the grain mother grain as a variable to study whether the grain maternal grain is in addition to the physicochemical characteristics and changes of the microorganisms in the mud, thus providing an application idea for the reuse of the grain mother. In the application research of microorganisms in the mud, Wang Mao separated three kinds of lactic acid bacteria: 
Lactobacillus penicillium, Lactobacillus breves and Pediococcus lactic. Gao Mingkun isolated 24 strains of Pediococcus from three kinds of Luzhou-flavor liquor. After identification by biochemical techniques, most of the Pediococcus were deemed to be be known, and two new Pediococcus were found $^{[3]}$. Domestic researchers have explored the construction and generation of microbial metabolic patterns from the mud, analyzed the metabolic characteristics of microorganisms in the mud, understanding the effects of metabolites on the quality of liquor, and the overall metabolic model of microorganisms in the mud. The fermentation mechanism of liquor makes a very important role.

\section{Study on important microorganisms of fragrant liquor}

At present, from the results of literature research, the research on important microorganisms in Luzhou-flavor and white-flavor liquor has numerous research results, and the research results of micro-organisms of fragrant liquor are less. Through inductive and combing, this paper finds that the research on microbes in fragrant liquor mainly focuses on the deductive law of microbes in the fermentation process and application research of Daqu quality. Lai Yucheng used alcohol as the research material to analyze the microbial deductive changes in the fermentation process ${ }^{[4]}$. Relevant researchers conducted in-depth research on microbes in the alcoholic Daqu, especially the comparison and induction of the three alcoholic songs of Red Heart, After Fire and Qingyu. In the specific fermentation production process and the storage changes of Daqu, the specifics of the mother song The amount of additional and related operational standards are discussed in detail. Lu Hui's research plays a major role in improving the fermentation quality and production process of alcohol abuse. Li Zengsheng also carried out detailed separation and identification of important microorganisms in the wine cellar during the fermentation process. The results showed that the essential microorganisms in the wine cellar of the fragrant liquor were mainly yeast, Candida and Pseudomonas; the bacteria mainly include lactic acid and Acetic acid bacteria; mold mainly includes Rhizopus, Monascus, Pythium and Mucor ${ }^{[5]}$.

\section{Analysis of important microorganisms in sauce-flavor liquor}

Sauce-flavored liquor focuses on the changes of related microbial groups and deductive rules in the production of sauce-flavored liquor, mainly concentrated in the application of production research, including the main functional bacteria of the sauce-flavor liquor and the characteristics of the wine body and the screening of related dominant strains, etc. In the research on the changes of main microbial groups and deductive regularity in the production of sauce-flavor liquor, domestic researchers have observed the microbial changes in microbial fermentation with dynamic and regional differences through the use of Langjiu as research materials, namely the microbial population has differences in quantity and quality in different levels of wine cellar. The overall pattern is that the upper layer is higher than the middle layer, and the inner layer is higher than the lower layer. Related bacillus and anaerobic bacteria are also higher in the upper layer than in the middle layer, and the middle layer is higher than the lower layer ${ }^{[6]}$. The research results of domestic researchers also include the temperature change during the accumulation process of the sauce-flavor liquor and the dynamic process of microbial changes. Therefore, in the production process of the sauce-flavor liquor, the dynamic changes and deductive laws of the microbial groups are influenced by the accumulation. Therefore, the temperature and time of the accumulation should be strictly controlled, so as to ensure the dynamic change of the microorganisms in the direction favorable to the fermentation of the wine. In the research on the characteristic components and functional bacteria of sauce-flavor liquor, the researchers found that they have strong protein active enzymes and hydrolase by separating the ring and bacillus siphonophore from the kanji. The results of the study indicate that the characteristic components in the wine are being co-fermented in the two bacilli. On the basis of this research, Li Xiangrong used the high-temperature stacked wine cellar of the sauce-flavored liquor as a material to isolate and isolate various strains of high-temperature and acid-resistant yeast strains. Through the fermentation capacity, the characteristics of the aroma and 
the analysis of secondary metabolites identification, thus determining that the functional bacteria of the sauce-flavor liquor are mainly $\mathrm{Y} 1$ and $\mathrm{Y} 5-\mathrm{-}^{[7]}$. Further, the morphological characteristics and physical and chemical properties of the principal functional bacteria were identified, which belonged to Italian yeast and terrestrial yeast respectively.

\section{Conclusions and prospects}

In this paper, we discuss the deductive changes of important microorganisms in different flavors of white wine, such as scent, aroma and sauce, and the growth and decline of fermentation, and have an overall understanding and overview of the research and application of important microorganisms in liquor.

Specifically, from the research direction, the research results of microorganisms in Luzhou-flavor liquor are the most extensive, while the results of fragrance and sauce are less. Different types of microbes have been lacking in research on bacteria in China. From the specific research content, the research on liquor microbes mainly includes the separation, purification and identification of microorganisms, the deductive changes and growth and decline of important microorganisms in liquor microbes, the screening and breeding of dominant functional strains in liquor microbes, etc. Also the research on related microorganisms in the fermentation environment of liquor production. From the research methods and technical analysis, the research on liquor microbes is in the stage of transition from traditional microbial research to molecular biotechnology, and it is gradually advanced and mature in research methods and application of physical and chemical technology. From the research level, the research on liquor microbes focuses on the practical application of production. The related genetic engineering, metabolic engineering and fermentation engineering theory are gradually deepening and systematic. The research on metabolic mechanism and stable process of microorganisms in the production process of liquor has just started. The charm of Chinese liquor fermentation is undergoing microbial evolution in solid state stationary fermentation, so the study of liquor microbes is of very important value. Looking forward to the future research should actively use advanced theories and methods to introduce bioengineering, metabolic engineering and micro-ecology and even molecular biology methods, in order to facilitate the disclosure of the microbial essence of liquor, thereby promoting the development and improvement of the liquor industry.

\section{References}

[1] Wang Peng, Wu Qun, Xu Yan. The relationship between the core microflora and environmental factors in Chinese liquor fermentation [J] Journal of Microbiology. 2018, 58(1): 142-153.

[2] Gao Ling, Xu Guansheng, Zhang Lei, Zeng Qinggu. Tracking study on main parameters of Luzhou-flavor liquor [J] Brewing technology 2018; 1: 47-48.

[3] Gao Mingkun, Wen Guangyu, Qian Fang. Research progress of microbial transformation and utilization of liquor grains [J] 2018,34(2): 191-193.

[4] Lai Yucheng. Producing multi-enzyme system mixed bacteria solid-state fermentation distiller's grains to produce protein feed [D] Fuzhou: Fujian Normal University, 2014:2-3.

[5] Lv Hui, Zhang Suyi, Feng Zhiping, et al. Study on Microbial Growth and Deterioration and Aroma Changes in Luzhou-flavor Liquor during Fermentation [J]. Food and Fermentation Technology,2015,46(3): 37-40.

[6] Liang Yanling, Su Fenfen, Wu Yanhua, et al. Study on the extraction of lactic acid from the yellow water of Daqu liquor by-product [J]. Brewing technology, 2017(3): 95-99.

[7] Li Xiangrong, Dai Xiaoqing, Wang Chunhui, et al. Extraction of microbial flocculant from excess sludge by resin method[J]. Journal of Central South University (Natural Science Edition) ,2013, 44(1): 411-416. 